\section{Kinetics study the decomposi- tion of the cellulose into cellulose nanocrystals by hydrothermal with hydrochloric acid catalyst}

\author{
Zulnazri Zulnazri, Rozanna Dewi, \\ Sulhatun Sulhatun Nasrun Nasrun \\ Chemical Engineering Department, \\ Malikussaleh University, Buket indah \\ Campus, Lhokseumawe, Aceh, \\ Indonesia
}

\begin{abstract}
The aim of this study was to hydrolyzed cellulose nanocrystals as cellulose-based biomass residues from oil palm by using hydrochloric acid under hydrothermal conditions. The characterization of cellulose nanocrystals was determined by FT-IR spectroscopy and $\mathrm{X}$ - ray diffraction. The infrared spectroscopy showed there has been a removal of lignin and hemicellulose in the spectrum. Crystallinity which reaches $78.59 \%$ was obtained by hydrolysis using hydrochloric acid catalyst $3 \mathrm{~mol} / \mathrm{L}$ with a reaction time of 1 hour. Based on the graph of $-\ln \quad \mathrm{C}_{\mathrm{A}} / \mathrm{C}_{\mathrm{A} 0}$ vs. time obtained that Cellulose nanocrystals forming reaction is of first order. The reaction rate constants to the formation of glucose $\left(\mathrm{k}_{2}\right)$ is greater than the reaction rate constant to the formation of Cellulose nanocrystals $\left(\mathrm{k}_{1}\right)$, which indicates that the phase of slow reaction is the reaction of the most influential on the overall reaction rate, the reaction of the formation of Cellulose nanocrystals.
\end{abstract}

\section{Introduction}

Biomass residue oil palm is abundant and renewable biomass in nature. Biomass is also called lignocellulosic waste because the main content is made by cellulose, hemicellulose and lignin. Cellulose is the molecule soluble in acid but does not easily dissolve in alkali, while the hemicellulose and lignin molecules soluble in alkali but not easily soluble in acid, so the hemicellulose and lignin easily removed from cellulose by using an alkali. ${ }^{1,2}$

Cellulose nanocrystals (CNCs) can be synthesized from cellulose using acid catalyst, wherein the acid initially degrades amorphous region of cellulose chain, further fragmentation by using sonication or hydrothermally to produce nanocrystals. Some common methods that have been used to synthesize CNCs are hydrolysis using sulfuric acid, unfortunately many crystalline cellulose is degraded, so that the yield obtained less than $35 \%, 3,4$ and the resulting product shows a low thermal stability. ${ }^{5-7}$ Hydrolysis of cotton pulp fibers by optimizing the preparation conditions using sulfuric acid can only increase the yield becomes $63.8 \%{ }^{8}$ Hydrolysis of cellulose-fiber sisal using $60 \% \mathrm{H}_{2} \mathrm{SO}_{4}$ can improve the crystallinity index nanocellulose to $72 \%$ and a particle size of $9.1 \mathrm{~nm}^{2}$ Some low concentration of mineral acids such as hydrochloric acid and maleic acid can be used to prevent aggregation and degradation of CNCs. ${ }^{9-12}$ Rosa and Correa reported that CNCs produced by hydrochloric acid hydrolysis showed high thermal stability, but that tends to provide liquid suspension flocculation and this can only be achieved with a low yield of $20 \% .{ }^{13,14}$ Filson and Andoh utilize high power ultrasonic irradiation with short periods of sonication have produced CNCs with a crystallinity of $78 \%$, but the yield achieved only 5\%. ${ }^{15}$ Tang et al. reported the hydrolysis method of cation-exchange resin can be applied to produce CNCs with the yield obtained $50.04 \%$ and reached $84.26 \%$ crystallinity, but the removal of the cationexchange resin with recurrent post-treatment can be time consuming chemical and many. ${ }^{16,17}$ According to Yu et al., the high yield and crystallinity is evident from the use of hydrochloric acid because the acid penetrates quickly into the deep layers of the network of cellulose and hydrolyze the amorphous regions chains of cellulose, while the crystalline regions are more resistant to acid hydrolysis lighter because the strength of hydrogen bonds between cellulose molecules adjacent compared with amorphous regions. Hydrolytic reaction was also limited by the relatively short reaction time so that the acid can only degrade amorphous regions in the cellulose. ${ }^{12}$ In this study, cellulose fibers hydrolyzed using hydrochloric acid to produce $\mathrm{CNCs}$ by hydrothermal processes. This process is more depth than the influence of the reaction time to the CNCs. Pursuant yield obtained $\mathrm{CNCs}$ do approach to the study of reaction kinetics in order to obtain the appropriate reaction order for this process.

\section{Materials and Methods}

\section{Materials}

Biomass residue oil palm was taken from crude palm oil processing plant. Chemical substance that was used for
Correspondence: Zulnazri Zulnazri, Department of Chemical Engineering, Sepuluh Nopember Institute of Technology, Keputih, Sukolilo, Surabaya 60111, East Java, Indonesia.

Te1.: +62.31.5994251 - Fax: +62.31.5943358. E-mail: zulnazri@unimal.ac.id

Key words: Cellulose, CNCs, hydrothermal, hydrochloric acid.

Acknowledgments: The author thank to Sepuluh Nopember Institute of Technology, Surabaya, Indonesia to facilitate this study.

Contributions: The author contributed equally.

Conflict of interest: The authors declare no potential conflict of interest.

Funding: none

Received for publication: 10 October 2017. Revision received: 13 December 2017.

Accepted for publication: 13 December 2017.

This work is licensed under a Creative Commons Attribution-NonCommercial 4.0 International License (CC BY-NC 4.0).

${ }^{\circ}$ Copyright: the Author(s), 2019

Licensee PAGEPress srl, Italy

International Journal of Plant Biology 2019; 10:7440 doi:10.4081/pb.2019.7440

residue oil palm extraction is $17,5 \% \mathrm{wt}$ $\mathrm{NaOH}$ from Merck and 2\% wt $\mathrm{NaOCl}$ were purchased from PT. Bratachem. Acid solution that was used for cellulose hydrolysis is hydrochloric acid $3 \mathrm{~mol} / \mathrm{L}$ from Merck. Aquadest that was used for washing the CNCs is taken from PT. Bratachem.

\section{Preparation of cellulose}

50 grams of dry weight residue oil palm was included in the $17.5 \%$ wt $\mathrm{NaOH}(1: 10)$ $\mathrm{w} / \mathrm{v}$, and then it was extracted at $80^{\circ} \mathrm{C}$ for 2 hours. Extract that obtained bleaching with $250 \mathrm{~mL}$ of $2 \%$ wt $\mathrm{NaOCl}$ at $70^{\circ} \mathrm{C}$ for 1 hour was also performed. Then, product obtained was filtered and washed until neutrals.

\section{Preparation of cellulose nanocrystals}

Cellulose 6:360 3M HCl (g:mL) was inserted in the ultrasonics bath type SU-27 $\mathrm{TH}$ capacity $477(\mathrm{~W}) \times 272(\mathrm{D}) \times 200(\mathrm{H})$ $\mathrm{mm}$, frequencies $28 \mathrm{kHz}$, output 300 watts and heater 500 watts, during 30 minutes. After that, sample hydrolyzed in the hydrothermal Parr USA pressure batch reactor, then the reactor tube was closed tightly and $\mathrm{N}_{2}$ gas was flowed into the reactor. The reaction was run at a temperature of $110^{\circ} \mathrm{C}$ with a reaction time of $0 ; 1 ; 2 ; 3 ; 4$ 
and 5 hours. After reaching the cellulosic suspension sampling time, the suspension obtained is added sufficient distilled water to stop the acid reaction, decanted in a bottle 1 to 2 days to precipitate particles of crystalline cellulose, and then washed with distilled water until neutral, centrifuged and dried.

\section{Characterization}

The chemical structures of OPEFB and the resulting cellulose were characterized on a Nicolet 8700 FT-IR spectrophotometer. FT-IR spectra were recorded in the spectral range of 4000-400 $\mathrm{cm}^{-1}$.

The crystal structures were characterized on a Philips PZ1200 X-ray difraction (X-RD) by using $\mathrm{Cu} \mathrm{K} \alpha \mathrm{X}$-rays with a voltage of $40 \mathrm{kV}$ and a current of $30 \mathrm{~mA}$. The crystallinity $(\mathrm{Crl})$ of the samples was determined by Segal's method. ${ }^{18}$

$$
\mathrm{Xc}=\frac{\left[I_{\text {(crstalline })}-I_{\text {(amoro })}\right]}{\mathrm{I}_{\text {(crystaline })}} \times 100 \%
$$

where $\mathrm{I}_{\text {(crystalline) }}$ is the intensity of the crystalline peak at the maximum at $2 \theta$ between $22^{\circ}$ and $23^{\circ}$ and $I_{(a m)}$ is the intensity at the minimum at $2 \theta$ between $18^{\circ}$ and $19^{\circ}$, where is the corresponding Bragg angle.

The crystallite sizes were estimated from the 110, 110, and 200 lattice planes of cellulose I by using the well-known Scherrer equation.

$$
\mathrm{D}_{h k l}=\frac{\mathrm{K} \cdot \lambda}{\mathrm{Bhkl} \cdot \cos \theta}
$$

where $\mathrm{D}_{h k l}$ is the crystallite size in the direction normal to the $h k l$ family of lattice planes, $K$ is the Scherrer constant (1.00 for equatorial reflections of rod-like or needlelike crystallites), $\lambda$ is the wavelength of the radiation $\left(1.54 \mathrm{~A}^{\circ}\right)$, and $B_{h k l}$ is the full width at half-maximum (FWHMLeft) in radians of the reflection of that family of lattice planes. $^{12}$

\section{Results}

\section{Condition preparation cellulose nanocrystals}

Supplementary Table S1 shown the yield, crystallinity and crystallite size of CNCs hydrolyzed using hydrochloric acid catalyst by hydrothermal process. All parameter using the ratio of cellulose-toacid $6 \mathrm{~g} / 360 \mathrm{~mL}$ with reaction temperature of $110^{\circ} \mathrm{C}$.

\section{Crystallinity}

Figure 1 shown the X-ray diffraction pattern of cellulose and CNCs prepared by hydrolysis of hydrochloric acid $3 \mathrm{~mol} / \mathrm{L}$. All the diffraction pattern for the cellulose crystals showed peaks around $2 \theta=16^{\circ}, 22^{\circ}$ and $35^{\circ}$, minimum diffraction pattern for amorphous plane showed around $2 \theta=18^{\circ}$ and $19^{\circ}$. Compared to cellulose, CNCs have broader peaks. It benefited as hydrochloric acid is more efficient in the removal of polysaccharides noncellulosic and dissolution of the amorphous zone, so CNCs have higher crystallinity. ${ }^{12}$

\section{Chemical structure}

The FT-IR spectrum in Figure 2 shown all the CNCs displaying a broad absorption peaks located at $3250-3500 \mathrm{~cm}^{-1}$ which is a stretch $-\mathrm{OH}$ group. The absorption peak at $2890-2920 \mathrm{~cm}^{-1}$ region associated with the group $-\mathrm{CH}_{2}$, while at the peak absorption 2890 and $2900 \mathrm{~cm}^{-1}$ is an overlapping of tape $-\mathrm{CH}_{2}$, this peak is only found in cellulosic feedstocks, while at the peak CNCs have been lost due to the amorphous cellulose chain termination.

\section{Kinetics of reaction}

Reaction series of hydrolysis of cellulose to CNCs and glucose is shown in below.

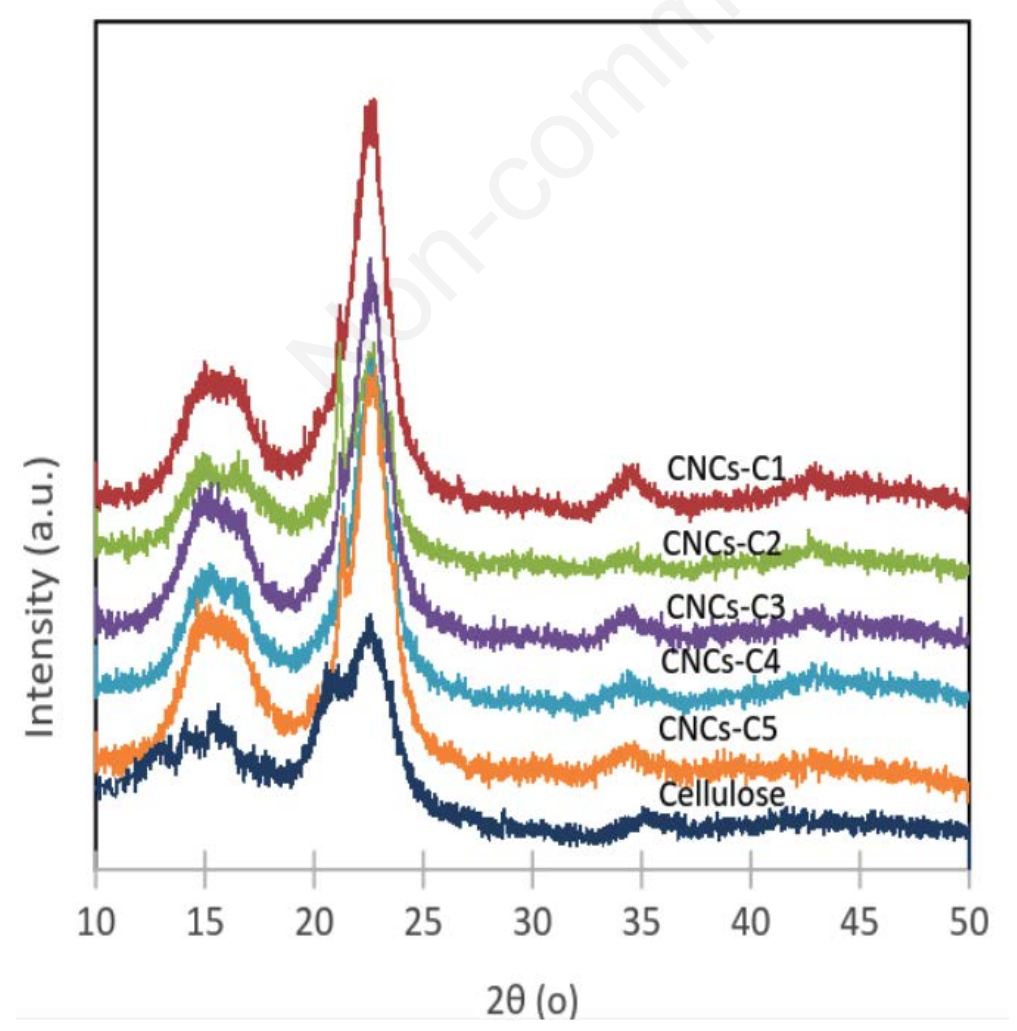

Figure 1. XRD of cellulose and CNCs are hydrolyzed with a reaction time of 1 to 5 hours.

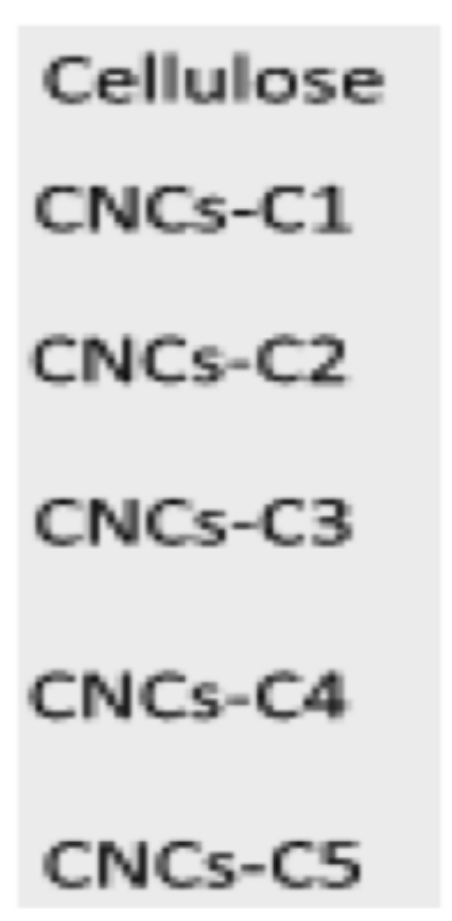

Figure 2. The FT-IR spectrum cellulose and CNCs are hydrolyzed with a reaction time of 1 to 5 hours. 


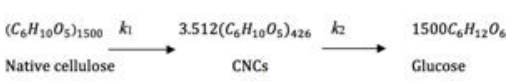

The above reaction can be written as:

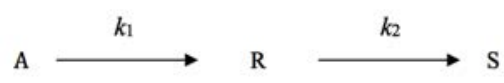

From the reactions known initial weight was $6 \mathrm{~g}$ cellulose, cellulose MW is 243,000 $\mathrm{g} / \mathrm{mole}, \mathrm{MW}$ CNCs is $69,000 \mathrm{~g} / \mathrm{mole}$ and MW glucose was $180.18 \mathrm{~g} / \mathrm{mole}$. As a reactant converted into product $\mathrm{R}$ is the yield $\mathrm{CNCs}$ and as product $\mathrm{S}$ is the concentration of glucose. Based on cellulose hydrolysis reaction is obtained kinetic model of the reaction.

The assumption of a homogeneous solution, the particles are small enough so that the resistance resulting from mass transfer can be ignored.

Stoichiometry for the concentration of reactants:

$\mathrm{C}_{\mathrm{A} 0}=\mathrm{CA}+\mathrm{C}_{\mathrm{R}}+\mathrm{C}_{\mathrm{S}}$

or $\mathrm{C}_{\mathrm{A}}=\mathrm{C}_{\mathrm{A} 0}-\mathrm{C}_{\mathrm{A} 0} \mathrm{X}$

To determine the value of $\mathrm{k}_{1}$ using the following equation:

$-\ln C_{\mathrm{A}} / C_{\mathrm{A} 0}=\mathrm{k}_{1} \mathrm{t}$, or $\mathrm{C}_{\mathrm{A}}=\mathrm{C}_{\mathrm{A} 0} e^{-\mathrm{k} 1 \mathrm{t}}$

If $\mathrm{k}_{2}$ is much greater than $\mathrm{k}_{1}$ :
$C_{\mathrm{S}}=C_{\mathrm{A} 0}\left(1-e^{-\mathrm{k} 1 \mathrm{t}}\right), \mathrm{k}_{2}>>\mathrm{k}_{1}$

The concentration of $\mathrm{R}$ can be calculated using the equation:

$$
\frac{C_{R \max }}{C_{A 0}}=\left(\frac{k_{1}}{k_{2}}\right)^{k_{2} /\left(k_{2}-k_{1}\right)}
$$

Based on the above equation can be determined the value of $C_{\mathrm{S}}, C_{\mathrm{R}}, C_{\mathrm{A}}$ and $-\mathrm{ln}$ $\mathrm{C}_{\mathrm{A}} / \mathrm{C}_{\mathrm{A} 0}$ as shown in Supplementary Table $\mathrm{S} 2$. From Supplementary Table S2 was obtained plot a graph of residual cellulose concentration $\left(C_{\mathrm{A}}\right)$ vs time, the concentration of CNCs $\left(C_{\mathrm{R}}\right)$ vs time and glucose concentration $\left(C_{\mathrm{S}}\right)$ vs time.

Figure 3 shows the concentration of cellulose $\left(C_{\mathrm{A}}\right)$ is reduced during the reaction, and the concentration of CNCs $\left(C_{\mathrm{R}}\right)$ increases until it reaches a peak on reaction time 1 hour, then decreased in line with the length of time the reaction is followed by increased concentrations of glucose $\left(C_{\mathrm{S}}\right)$ along the length of reaction time, so that the reaction is considered to be a series reaction. Decreased in concentration CNCs $\left(C_{\mathrm{R}}\right)$ due to excessive degradation of crystalline cellulose so that increased concentrations of glucose $\left(C_{\mathrm{S}}\right)$, so that the reaction time becomes the main influential factor in the study of the kinetics of hydrolysis of cellulose into CNCs. The graph above may not be combined because of differences in

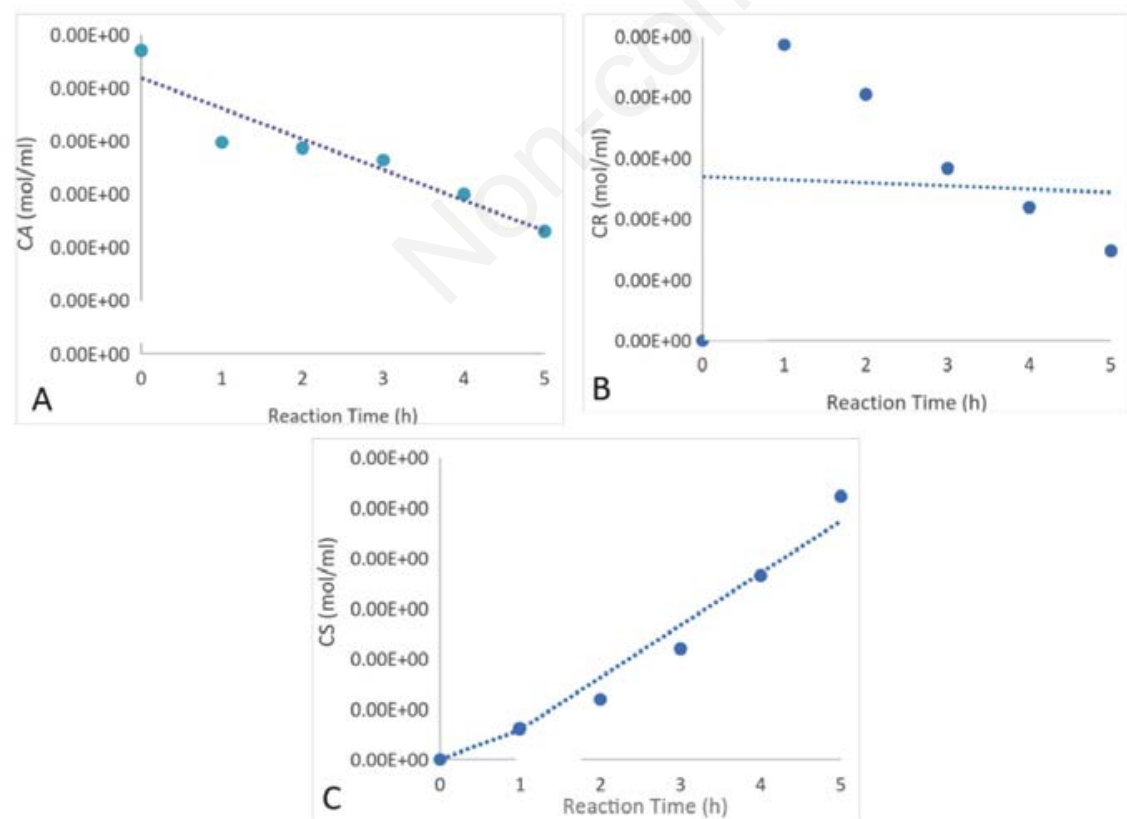

Figure 3. Relationship between (A) the concentration of residual cellulose (CA) vs time, (B) the concentration of CNCs (CR) vs time and (c) the concentration of glucose (CS) vs time. scale.

Figure 4 is a graph of $-\ln C_{\mathrm{A}} / C_{\mathrm{A} 0}$ vs time obtained from the calculation of the concentration of the remainder of the initial concentrations based on data from Supplementary Table S2. Figure 4 strengthen the evidence that the longer the reaction time, the remaining reactants concentration is getting smaller, where $-\ln C_{\mathrm{A}} / C_{\mathrm{A} 0}$ obtained increasing together with the increasing reaction time, so this graph reflects the equation of order 1 .

Figure 5 can be seen experimental glucose concentration ( $C_{\mathrm{S}}$ experiment) higher than the concentration of glucose models $\left(C_{\mathrm{S}}\right.$ model $)$, it indicates that the formation of glucose can occur directly from the decomposition of the cellulose without via formation of CNCs.

\section{Discussion}

\section{Condition preparation cellulose nanocrystals}

Figure 6 shows the longer the reaction time, the yield obtained tends to be down, this is due to long reaction times hydrochloric acid can attack the cellulose crystalline regions thus reducing the crystallinity of CNCs. According to $\mathrm{Yu}$ et al., hydrolytic reaction is limited by the relatively short reaction time so that the acid can only be lowered amorphous regions in the cellulose and leave the crystal region. So that the reaction time is believed to be one of the most important parameters to be considered to improve the quality of CNCs. ${ }^{12}$

\section{Crystallinity}

Crystallinity $\mathrm{CNCs}-\mathrm{C} 1$ which reaches $78.59 \%$ believed that the hydrolysis reaction using hydrochloric acid catalyst in a reaction time of 1 hour can help up to

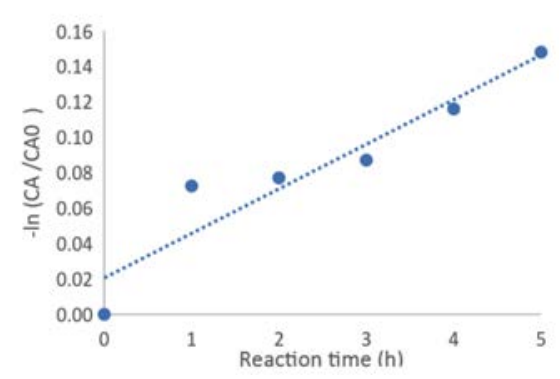

Figure 4. Relationship between $-\ln \mathrm{C}_{\mathrm{A}} / \mathrm{C}_{\mathrm{A} 0}$ vs time. 


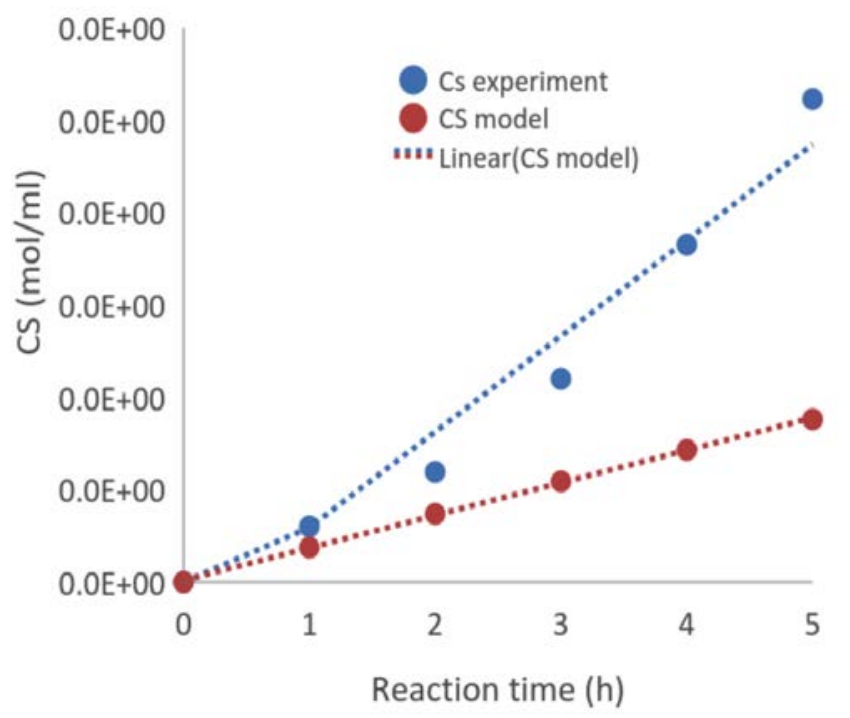

Figure 5. Relationship between glucose concentration (Cs) vs time.

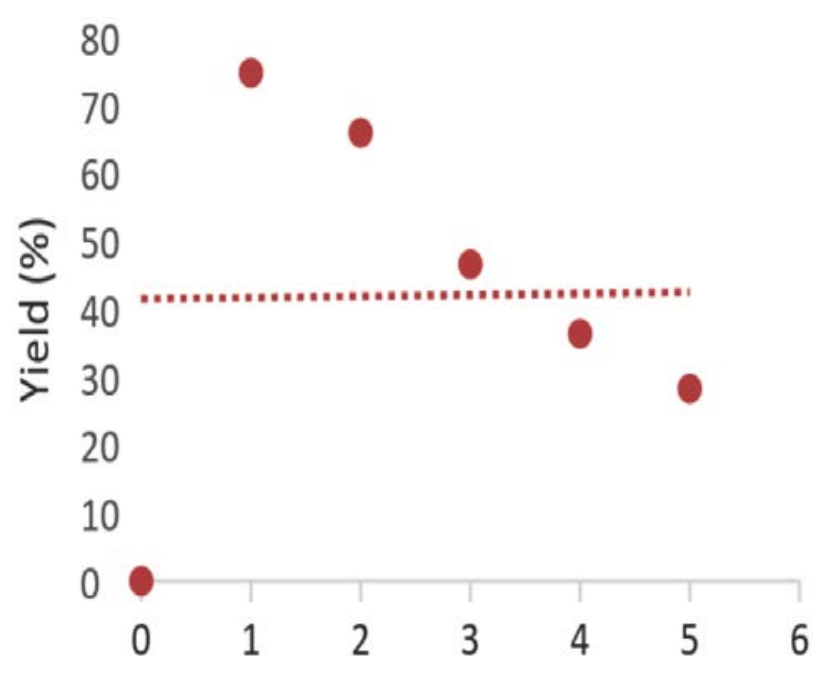

Figure 6. Graph relationship yield of CNCs vs reaction time. removal amorphous cellulose chains. Furthermore, the crystallinity declined slowly with increasing reaction time, as seen in the CNCs-C2 fell to $77.07 \%$ and CNCs-C5 with a longer reaction time dropped further to $73.27 \%$ This is due to the long time of the hydrolysis reaction may decide fraction of crystalline regions of cellulose so degraded into simpler chain. Hydrochloric acid hydrolysis with reaction time is too long can weaken the cellulose crystalline regions, even resulting in carbonization CNCs. ${ }^{12}$

CNCs shown maximum crystal diffraction peaks located at the angle $2 \theta=22.6^{\circ} \pm$ 0.2 with 002 lattice planes, which shows crystalline cellulose I. ${ }^{19,20}$ At an angle $2 \theta=$ $35^{\circ}$ all diffraction pattern still shows a peak crystalline cellulose are low, this indicates that hydrochloric acid catalyst capable of persevering region crystals that are at the peak of the weak areas. ${ }^{19}$ Overall CNCs shows the crystallite size below $7.83 \mathrm{~nm}$, this proves that the hydrolysis using acid catalysts are applied can reduce the size of crystalline cellulose.

\section{Chemical structure}

Absorption in the $1600-1650 \mathrm{~cm}^{-1}$ in the cellulose samples is an indication of the absorption of water or stretch $\mathrm{O}-\mathrm{H}$, in the spectrum absorption peaks are already getting smaller this is because the crystal structure is already happening removal of water. According to Johar et al., this peak indicates the bending form water molecules due to a strong interaction between cellulose and water. ${ }^{21}$ Absorption peaks in the region $1300-1365 \mathrm{~cm}^{-1}$ in all samples a ribbon of vibration of $\mathrm{C}-\mathrm{H}$ and $\mathrm{C}-\mathrm{O}$ associated with aromatic rings polysaccharides, ${ }^{19}$ this absorption band according to data analyzed by the Nacos. ${ }^{22}$ That looks sharp absorption peaks at $1000-1160 \mathrm{~cm}^{-1}$ caused by the deformation of vibration of C-H. According to Kagarzadeh et al., peak is a vibration deformations $\mathrm{C}-\mathrm{H}$ and pyranose $\mathrm{C}-\mathrm{O}-\mathrm{C}$ is more prevalent. $^{23}$ Spectrum $\mathrm{CNCs}$ also shows the intensity has increased at the peak, it implies an increase in the value of crystalline cellulose. ${ }^{14}$

\section{Kinetics of reaction}

CNCs forming reaction rate constant $\left(k_{1}\right)$ is obtained from the graph in Figure 6. Glucose forming reaction rate constants $\left(\mathrm{k}_{2}\right)$ is obtained from the substitution $C_{\mathrm{R}}$ values into the equation (4), where $C_{R}$ obtained from the Supplementary Table S2. From graph, value of $\mathrm{k}_{1}$ obtained is $0.0252 \mathrm{~h}^{-1}$ and the value $\mathrm{k}_{2}$ that obtained based calculation is $3,454 \mathrm{~h}^{-1}$. The reaction rate constant in the formation of glucose $\left(\mathrm{k}_{2}\right)$ is greater than the reaction rate constant to the formation of CNCs $\left(\mathrm{k}_{1}\right)$. This is consistent with the study of Wang et al., who reported that the constant formation reaction of glucose faster than $\mathrm{CNCs}^{24}$ Value $\mathrm{k}_{2}>\mathrm{k}_{1}$ indicates that the slowest reaction stage is the stage of reaction greatest influence on the overall rate of reaction is the reaction of the cellulose into CNCs.

\section{Conclusions}

The concentration of cellulose $\left(C_{A}\right)$ is reduced during the reaction time, while the concentration of CNCs $\left(C_{R}\right)$ increases until it reaches a peak on reaction time 1 hour, and then decreased in line with the length of time the reaction is due to the increasing concentration of glucose $\left(C_{S}\right)$, so that the reaction is considered to be a series reaction. The longer the reaction time, the concentration of the reactants is left is getting smaller, which - $\ln C_{A} / C_{A 0}$ gained increasing along with the increasing reaction time, so this graph reflects the first order equations. Based on the comparison of the graphic (Cs) experiment with (Cs) model of the formation of glucose can occur directly from the decomposition of cellulose.

\section{References}

1. Gherbin P, Simone M, Giuseppe M, Antonio S. Shooting of giant reed (Arundo donax L.) stem cuttings in cold greenhouse. Int J Plant Biol 2016;7:315.

2. Moran JI, Alvarez VA, Cyras VP, Vazquez A. Extraction of cellulose and preparation of nanocellulose from sisal fibers. Cellulose 2008;15:149-59.

3. Bondeson D, Mathew A, Oksman K. Optimization of the isolation of nanocrystals from microcrystalline cellulose by acid hydrolysis. Cellulose 2006;13:171-80.

4. Huyly T, Kasen S. Bioformulations and nano product from Chaetomium cupreum CC3003 to control leaf spot of rice var. Sen Pidoa in Cambodia. Int $\mathrm{J}$ Plant Biol 2016;7:59-63.

5. Azouz KB, Ramires EC, Fonteyne WV, et al. Simple method for the melt extrusion of a cellulose nanocrystal rein- 
forced hydrophobic polymer. ACS Macro Lett 2012;1:236-40.

6. Bondeson D, Oksman K. Polylactic acid/cellulose whisker nanocomposites modified by polyvinyl alcohol. Appl Sci Manufact 2007;38:2486-92.

7. Oksman K, Mathew AP, Bondeson D, Kvien. Manufacturing process of cellulose whiskers/polylactic acid nanocomposites. Composite Sci Tech 2006;66:2776-84.

8. Fan J, Li Y. Maximizing the yield of nanocrystalline cellulose from cotton pulp fiber. Carbohydrate Polymers 2012;88:1184-8.

9. Jiang F, Esker AR, Roman M. Acid-catalyzed and solvolytic desulfation of H2SO4-hydrolyzed cellulose nanocrystals. Langmuir 2010;26:17919-25.

10. Chen W, Yu H, Liu Y, et al. Individualization of cellulose nanofibers from wood using high-intensity ultrasonication combined with chemical pretreatments. Carbohyd Polym 2011;83:1804-11.

11. Tang L, Huang B, Lu Q, et al. Ultrasonication-assisted manufacture of cellulose nanocrystals esterified with acetic acid. Bioresour. Techno 2013;127:100-5.

12. Yu H, Qin Z, Liang B, et al. Facile extraction of thermally stable cellulose nanocrystals with a high yield of $93 \%$ through hydrochloric acid hydrolysis under hydrothermal conditions. J Mater Chem A 2013;1:3938-44.

13. Rosa MF, Medeiros ES, Malmonge JAK, et al. Cellulose nanowhiskers from coconut husk fibers: effect of preparation conditions on their thermal and morphological behavior. Carbohyd Polym 2010;81:83-92.

14. Correa AC, Teixeira EM, Pessan LA, Mattoso LCH. Cellulose nanofibers from curaua fibers. Cellulose 2010;17:1183-92.

15. Filson PB, Dawson-Andoh BE. Sonochemical preparation of cellulose nanocrystals from lignocellulose derived materials. Biores Technol 2009; 100:2259-64.

16. Tang L, Huang B, Ou W, et al. Manufacture of cellulose nanocrystals by cation exchange resin-catalyzed hydrolysis of cellulose. Bioresour Technology 2011;102:10973-7.

17. Carina B, Rul MAM, Isabel AP, et al. Effects of substrate type on plant growth and nitrogen and nitrate concentration in spinach. Int $\mathrm{J}$ Plant Biol 2016;7:44-7.

18. Seagal L, Creely JJ, Martin Jr AE, Conrad CM. An empirical method for estimating the degree of crystallinity of native cellulose using the $\mathrm{x}$-ray diffractometer. Text Res J 1959;29:786-94.
19. Zulnazri, Roesyadi A, Sumarno. Effects of hydrolysis conditions on the crystallinity, chemical structure, morphology, and thermal stability of cellulose nanocrystals extracted from oil palm biomass residue. Int $\mathrm{J}$ Chem Tech Res 2016;9:456-64.

20. Sangian HF, Kristian J, Rahma S, et al. Comparative study of the preparation of reducing sugars hydrolyzed from highlignin lignocellulose pretreated with ionic liquid, alkaline solution and their combination. J Eng Technol Sci 2015;47:137-48.

21. Johar N, Ahmad I, Dufresne A. Extraction, preparation and characterization of cellulose fibres and nanocrystals from rice husk. Industr Crops Prod 2012;37:93-9.

22. Nacos M, Katapodis P, Parppas C, et al. A source of biologically active acidic oligosaccharides. Christakopoulos Carbohyd Polym. 2006;66:126-34.

23. Kagarzadeh H, Ahmad I, Abdullah I, et al. Effects of hydrolysis condition on the morphology, crystalinity and thermal stability of cellulose nanocrystals extracted from kenal bast fibers. Cellulose 2012;19:855-66.

24. Wang N, Ding E, Cheng R. Effects of mechanical fibrillation on cellulose reinforced poly (ethylene oxide). Polymer 2007;48:3486-93. 